\title{
Epigenetic Silencing of Somatostatin in Gastric Cancer
}

\author{
Kaya Jackson ${ }^{1, \#, ~ M o h a m m e d ~ S o u t t o ~}{ }^{2, \#}$, DunFa Peng ${ }^{2, \#}$, TianLing $\mathrm{Hu}^{2}$, Dana Marshal ${ }^{1}$, and \\ Wael El-Rifai $2,3,4,{ }^{*}$ \\ 1 Department of Surgery, Meharry Medical College, Nashville, Tennessee, USA \\ 2 Department of Surgery, Vanderbilt University Medical Center, Nashville, Tennessee, USA \\ ${ }^{3}$ Department of Cancer Biology, Vanderbilt University Medical Center, Nashville, Tennessee, \\ USA \\ ${ }^{4}$ Vanderbilt-Ingram Cancer Center, Vanderbilt University Medical Center, Nashville, Tennessee, \\ USA
}

\begin{abstract}
Background-Somatostatin (SST), a primary inhibitor of gastrin-stimulated gastric acid secretion, has potent antitumor and anti-secretory activities in several human cancers.
\end{abstract}

Aims-This study was performed to investigate the SST gene expression levels and possible epigenetic mechanisms that regulate its expression in gastric adenocarcinomas.

Methods-Quantitative real time-RT PCR and quantitative bisulfite pyrosequencing technologies were applied to study primary gastric cancer tissue samples and cell lines.

Results-Quantitative real-time RT-PCR analysis demonstrated down-regulation of SST transcript in $93 \%$ of gastric carcinoma samples (30/32), as compared to 21 normal samples $(P<0.001)$. Because of the presence of a large $\mathrm{CpG}$ island in the SST promoter, we next examined its promoter DNA methylation levels using quantitative bisulfite pyrosequencing technology. The results demonstrated a significant increase in SST promoter DNA methylation levels in tumor samples as compared to normal samples $(P<0.05)$. Promoter DNA hypermethylation and silencing of SST was also detected in seven gastric cancer cell lines that we tested. To confirm the role of promoter DNA methylation as an epigenetic mechanism regulating SST expression, AGS gastric cancer cells were treated with 5-Aza-deoxycytidine. This treatment led to reduction in the promoter DNA methylation levels of SST accompanied by restoration of its mRNA expression.

Conclusions-Our results indicate that promoter DNA methylation levels play a critical role in regulating SST expression in gastric cancer. This finding provides a foundation for further studies on the role of SST in gastric carcinogenesis and its potential as a biomarker for gastric cancers.

\section{Keywords}

methylation; expression; somatostatin; gastric cancer

\section{Introduction}

Gastric adenocarcinoma remains the fourth most common cancer and the second leading cause of cancer death worldwide [1]. The two main tumor sites of gastric cancer are the

\footnotetext{
"Correspondence should be addressed to: Wael El-Rifai, M.D., Ph.D., Vanderbilt University Medical Center, 1255 Light Hall, 2215 Garland Avenue, Nashville, TN 37232, wael.el-rifai@ vanderbilt.edu.

\# contributed equally
} 
proximal and distal stomach. Despite a decline in distal (non-cardia) gastric cancers, proximal (cardia) gastric cancers have been increasing in incidence in the Western countries $[1,2]$. The main risk factors for distal gastric cancer include Helicobacter pylori infection and dietary factors including nitrate intake, excess of salt and a deficiency in fresh fruit and vegetables, whereas gastroesophageal reflux disease and obesity are important factors in the development of proximal gastric cancer [1]. Gastric cancer remains a disease of poor prognosis and high mortality [3-5].

Epigenetically mediated transcriptional silencing events such as increases in DNA methylation regulate important cell functions in all stages of tumor formation and progression [6,7]. DNA methylation levels can be useful in assessing cancer risk, early detection, prognosis and prediction of response to cancer therapy $[7,8]$. Somatostatin (SST), a peptide hormone, is secreted by the gastrointestinal tract, pancreas and areas of the central nervous system [9]. Its main functions are to regulate endocrine and exocrine secretion, modulate motor activity, and inhibit gastrin-stimulated gastric acid secretion in the gastrointestinal tract [9]. Several in vitro and in vivo studies have suggested that SST functions as a tumor suppressor gene in human cancers [9]. SST is known to suppress tumor growth through distinct mechanisms that involve inhibition of growth factors and hormones, reduction in the vascularization, and regulation of the immune system [10-12]. Stable analogs of SST are widely used in treating neuroendocrine tumors because they are effective in decreasing hormone secretion[10] [11]. In the gastrointestinal tract, SST is a paracrine transmitter, where it is normally released from D cells and acts on adjacent or nearby cells to exert its inhibitor effects $[10,11]$. In certain tumors, however, SST operates through an autocrine mechanism to suppress tumor growth. In this manner, SST is both released from and acts on the tumor itself primarily through SST receptors type 2 [10,11].

In this study, we have investigated the expression of SST in gastric cancer and utilized quantitative bisulfite pyrosequencing technology (Biotage, Uppsala, Sweden) for analysis of its promoter DNA methylation.

\section{Materials and Methods}

\section{Tissues samples}

All tissue samples were obtained from the archives of pathology at Vanderbilt University (Nashville, TN, USA), and from the National Cancer Institute Cooperative Human Tissue Network (CHTN). All personal identifiers were removed prior to receiving samples. The use of specimens from the archival tissue repository was approved by the Institutional Review Board protocol. All tissue samples included in this study were coded and collected from tissues that remained after completion of diagnosis, and that are otherwise discarded. All tumors and normal gastric mucosal epithelial tissues were histologically verified. The gastric adenocarcinomas were distal (antrum and body) and ranged from well-differentiated (WD) to poorly-differentiated (PD), stages I to IV, with a mix of intestinal and diffuse-type tumors.

\section{Quantitative real-time reverse transcription PCR (qRT-PCR) analysis of SST-} Total RNA was isolated using the RNeasy mini kit (Qiagen, Valencia, CA, USA), and single-stranded cDNA was subsequently synthesized using the Advantage RT-for-PCR Kit (Clontech, Palo Alto, CA, USA). The expression of SST was evaluated in a set of 53 frozen primary human samples that included 21 normal mucosa of the stomach and 32 samples of gastric carcinoma. Fourteen samples had a matching tumor and adjacent normal sample from the same patient. The SST oligos (forward 5'-AGAAGTCCCTGGCTGCTG-3', and reverse 5'-GTTCCAGGGCATCATTCTCC-3') were designed using the online software, Primer 3 (http://frodo.wi.mit.edu/cgi-bin/primer3/primer3_www.cgi). The qRT-PCR was 
performed using an iCycler (Bio-Rad, Hercules, CA, USA), with the threshold cycle number determined by use of iCycler software version 3.0. Reactions were performed in triplicates and the threshold numbers were averaged. The results of the SST gene were normalized to $H P R T$ (forward 5'-TTGGAAAGGGTGTTTATTCCTCA-3', and reverse 5'-

TCCAGCAGGTCAGCAAAGAA-3'), which had minimal variation in all normal and tumor samples tested, and is therefore considered to be a reliable and stable reference gene for realtime RT-PCR. Expression fold was calculated according to the formula $2^{(R t-E t)} / 2^{(R n-E n)}$ [13] where $R t$ is the threshold cycle number for the reference gene (HPRT) observed in the tumor, $E t$ is the threshold cycle number for the experimental gene (SST) observed in the tumor, $R n$ is the threshold cycle number for the reference gene observed in the normal samples, and $E n$ is the threshold cycle number for the reference gene observed in the normal. $R n$ and $E n$ values were calculated as an average of the 21 normal samples. For all the primary gastric carcinoma samples, the gene was considered to be down-regulated if the mRNA expression fold was $\leq 0.5$ in comparison with the normal samples.

DNA Bisulfite treatment and pyrosequencing analysis-DNA was purified using a DNeasy tissue kit (Qiagen). The bisulfite modification of the DNA from cell lines and tissues was performed using an EZ DNA Methylation-Gold Kit (ZYMO Research, Orange, CA, USA), according to the manufacturer's protocol. The SST promoter CpG Island (from -83 to +678 bp from the transcription start site) was identified using a $\mathrm{CpG}$ island online search tool (http://www.uscnorris.com/cpgislands2.cpg.aspx). The criteria used for the definition of $\mathrm{CpG}$ islands was; a DNA fragment $\geq 500 \mathrm{bp}$ with a $\mathrm{G}+\mathrm{C}$ equal to or greater than 55\% with an observed $\mathrm{CpG} /$ expected $\mathrm{CpG}$ of 0.65. A $40 \mathrm{ng}$ aliquot of modified DNA was subjected to PCR amplification of the specific promoter region containing the $\mathrm{CpG}$ island. The primers were designed using PSQ assay design software (Biotage), where one of the primers was biotin labeled. The forward primer sequence is

AGGGAGAAGGTTGAGAGTATATAA, the reverse biotin labeled primer sequence is BioAAAAACTAACCAAATCTAAAATCC and the sequence primer is

GAAGGTTGAGAGTATATAAG. The Platinum PCR SuperMix High Fidelity enzyme mix (Invitrogen, Carlsbad, CA, USA) was used in the PCR reactions. The PCR products were checked by gel electrophoresis to confirm the size of the product and rule out the formation of primer dimers. The specific PCR products were then subjected to quantitative pyrosequencing analysis using a Biotage PyroMark MD system (Biotage) following the protocol provided by the manufacturer. The results were analysed by Pyro Q-CpG 1.0.9 software (Biotage). The percent of methylation levels in the normal samples were compared to those in tumor samples for identification of promoter DNA hypermethylation of SST in tumor samples. Statistical analysis was performed to detect significant changes in the frequencies of DNA methylation of $\mathrm{CpG}$ sites between tumor and normal samples.

\section{5-Aza-2' deoxycytidine (5-Aza) and trichostatin-A (TSA) treatment-For} validation of the role of promoter DNA hypermethylation in transcriptional regulation of SST in vitro, AGS gastric cancer cell line was used. AGS cells were maintained in Dulbecco's modified Eagle's medium (DMEM), supplemented with $10 \%$ fetal bovine serum (FBS) and antibiotics (Invitrogen). Cells were seeded at low density for $24 \mathrm{~h}$, and then treated with $5 \mu \mathrm{M}$ 5-Aza (Sigma-Aldrich, St Louis, MO, USA) for $72 \mathrm{~h}$ or $300 \mathrm{nM}$ Trichostatin A (TSA, Wako, Osaka, Japan) for 24h. Total RNA and DNA were isolated and purified by RNeasy kit and DNeasy tissue kit (Qiagen), as described above. DNA methylation levels of the $\mathrm{CpG}$ nucleotides of SST promoter were determined by pyrosequencing before and after treatments. The SST mRNA expression levels were determined by qRT-PCR, as described above. 
Statistical Analysis-Statistical significance of two groups was determined by Student's t-test using GraphPad prism version 4 software (GraphPad Prism Software, Inc., La Jolla, CA, USA). The student's t-test was used to compare the differences of the DNA methylation level between normal and gastric carcinoma, the differences of mRNA expression fold between normal and gastric carcinoma, and the differences of mRNA expression fold between unmethylated and methylated gastric adenocarcinoma. The correlation between the DNA methylation level and mRNA expression fold was determined by Spearman correlation. All $p$ values were based on two-sided tests, and the differences were considered statistically significant when the $p$ value was $\leq 0.05$.

\section{Results}

\section{Down-regulation of SST mRNA expression in gastric carcinoma}

The qRT-PCR analysis showed frequent down-regulation of SST mRNA expression in primary gastric carcinoma samples (30/32, 93.8\%), as compared to 21 normal gastric mucosa samples $(p<0.001)$ (Figure 1A). Analysis of 14 paired tumor and normal samples further demonstrated significant $(p<0.01)$ down-regulation of SST mRNA expression in tumors compared to their corresponding normal samples (Figure 1B).

\section{Promoter DNA hypermethylation of SST gene correlates with silencing of mRNA expression}

Quantitative analysis of SST promoter DNA methylation using state-of-the-art pyrosequencing technology indicated a significant increase in promoter DNA methylation levels in tumor samples, as compared to normal samples $(P<0.05)$. The representative results of the percentage of methylation for the SST gene promoter in gastric carcinoma versus normal are shown in Figure 2A. Analysis of DNA methylation in 12 tumor samples demonstrated a significant increase in the level of DNA methylation as compared to their adjacent histologically normal samples from the same patients $(p<0.01)$. A representative summary is illustrated in Figure 2B. We further analyzed the promoter DNA methylation against mRNA expression levels in 13 samples where both DNA and RNA were available. Using spearman correlation for analysis we found a significant inverse correlation between promoter DNA methylation and mRNA expression fold for SST (coefficient $r=-0.56$, $p=0.02$ ) (Figure 3). These results suggest that the hypermethylation of the SST promoter region is one of the factors involved in suppression of its mRNA expression in gastric carcinomas.

\section{Treatment with 5-aza-2'-deoxycytidine alone or combined with trichostatin A results in activation of SST gene expression}

To confirm the role of DNA methylation in silencing SST expression, we measured the promoter DNA hypermethylation of the SST gene in seven gastric cancer cell lines (AGS, MKN28, MKN45, MKN75, KATOIII, SNU1, and SNU16); all originated form distal gastric locations (antrum or body). All gastric showed more than 50\% promoter DNA methylation levels (Figure 4A). Of note, these seven gastric cancer cell lines demonstrated complete loss of SST expression. We next used the AGS gastric cancer cells as an in vitro model to determine whether interference with the activities of DNA methyltransferases lead to reactivation of SST expression. The AGS cells had no detectable level of SST mRNA and their promoter demonstrated 95\% DNA methylation. The 5-Aza treatment led to reduction in the DNA methylation from 95 to $76 \%$ and re-expression of SST. The combination of 5Aza and TSA treatment significantly reduced the DNA methylation from 95 to $43 \%$ and demonstrated a synergistic potent increase in mRNA expression confirming the epigenetic silencing of SST (Figure 4B). 


\section{Discussion}

In this study, we have shown that SST mRNA expression is frequently down-regulated in primary gastric cancer samples. Using state-of-the-art pyrosequencing technology for quantitative analysis of promoter DNA methylation of SST, we detected an increased percentage of DNA methylation levels in gastric adenocarcinomas as compared to normal gastric mucosa samples. Concordant with the primary tissue samples, the seven gastric cancer cell lines that we tested lacked the expression of SST and showed high levels of DNA methylation. These results suggested that epigenetic mechanisms could be the leading cause of silencing SST expression in gastric cancer. DNA hypermethylation and loss of expression of SST has been shown in colon and esophageal cancer $[9,10]$. Taken together, silencing of SST could be a critical step in gastrointestinal tract carcinogenesis irrespective of the anatomical tumor site.

Using AGS gastric cancer cells as an in vitro model for validation of the impact of DNA methylation on SST expression, we showed that treatment with 5-Aza-dc alone led to reexpression of low levels of SST. The combined treatment with 5-Aza-dc and TSA had a synergistic effect on restoration of SST expression. Therefore, both DNA methyl transferase inhibitors and histone deacetylase inhibitors were required for the maximum restoration of SST expression, consistent with the interpretation that silencing of SST in gastric cancer is mediated by epigenetic mechanisms that include DNA hypermethylation and histone deacetylation. A similar synergism following a combination of 5-Aza-dc and TSA was reported in the reactivation of epigenetically silenced tumor suppressor genes [14]. Interestingly, we observed that TSA administration following 5-Aza-dc treatment enhanced the DNA demethylation of SST. These results are concordant with recent studies that suggested that TSA can have a demethylation effect in a gene-specific manner $[15,16]$. This effect could be mediated by down-regulation of DNA methyltransferase 3b (DNMT3b) [17] or DNMT1 [18]. However, the detailed mechanism by which TSA increases the demethylation effect of 5-Aza-dc remains unclear and requires further investigation.

Aberrant DNA hypermethylation of gene promoter regions is an important epigenetic mechanism that regulates gene expression leading to downregulation and silencing of several tumor suppressor genes [19-24]. Several in vitro and in vivo studies have suggested that SST functions as a tumor suppressor gene in human cancers [9] that can inhibit tumor growth through mechanisms that involve inhibition of growth factors and hormones [11,12] and reduction in the vascularization [11,12]. Silencing of SST in gastric cancer suggests that one or more of the aforementioned mechanisms could be mediated by the loss of SST during gastric carcinogenesis. However, additional studies to document the biological outcome of loss of SST in gastric carcinogenesis are needed.

In summary, this study demonstrates frequent epigenetic regulation of SST expression in gastric cancer. This finding provides a foundation for further studies on the role of SST in gastric carcinogenesis and its potential as a biomarker for early diagnosis, therapy and prognosis of gastric cancers.

\section{Acknowledgments}

This study was supported by the National Institute of Health grants R01CA93999 and R25CA102209. The contents of this work are solely the responsibility of the authors and do not necessarily represent the official views of the National Cancer Institute or Vanderbilt University. 


\section{References}

1. Crew KD, Neugut AI. Epidemiology of gastric cancer. World J Gastroenterol. 2006; 12:354-362. [PubMed: 16489633]

2. Hansson LE, Sparen P, Nyren O. Increasing incidence of carcinoma of the gastric cardia in sweden from 1970 to 1985. Br J Surg. 1993; 80:374-377. [PubMed: 8472157]

3. Roder DM. The epidemiology of gastric cancer. Gastric Cancer. 2002; 5 (Suppl 1):5-11. [PubMed: 12772880]

4. Hohenberger P, Gretschel S. Gastric cancer. Lancet. 2003; 362:305-315. [PubMed: 12892963]

5. Berardi R, Scartozzi M, Galizia E, Cascinu S. Adjuvant chemotherapy in gastric cancer. The italian experience and review of the literature. Suppl Tumori. 2003; 2:S45-47. [PubMed: 12914390]

6. Jones PA. Epigenetics in carcinogenesis and cancer prevention. Ann N Y Acad Sci. 2003; 983:213219. [PubMed: 12724226]

7. Baylin SB, Ohm JE. Epigenetic gene silencing in cancer - a mechanism for early oncogenic pathway addiction? Nat Rev Cancer. 2006; 6:107-116. [PubMed: 16491070]

8. Jones PA, Baylin SB. The fundamental role of epigenetic events in cancer. Nat Rev Genet. 2002; 3:415-428. [PubMed: 12042769]

9. Jin Z, Mori Y, Hamilton JP, Olaru A, Sato F, Yang J, Ito T, Kan T, Agarwal R, Meltzer SJ. Hypermethylation of the somatostatin promoter is a common, early event in human esophageal carcinogenesis. Cancer. 2008; 112:43-49. [PubMed: 17999418]

10. Mori Y, Cai K, Cheng Y, Wang S, Paun B, Hamilton JP, Jin Z, Sato F, Berki AT, Kan T, Ito T, Mantzur C, Abraham JM, Meltzer SJ. A genome-wide search identifies epigenetic silencing of somatostatin, tachykinin-1, and 5 other genes in colon cancer. Gastroenterology. 2006; 131:797808. [PubMed: 16952549]

11. Liddle RA, Jirtle RL. Epigenetic silencing of genes in human colon cancer. Gastroenterology. 2006; 131:960-962. [PubMed: 16952566]

12. Reubi JC, Laissue JA. Multiple actions of somatostatin in neoplastic disease. Trends in pharmacological sciences. 1995; 16:110-115. [PubMed: 7792931]

13. El-Rifai W, Moskaluk CA, Abdrabbo M, Harper JC, Yoshida C, Riggins G, Frierson HF Jr, Powell SM. Gastric cancers overexpress s100a calcium binding proteins. Cancer Res. 2002; 62:68236826. [PubMed: 12460893]

14. Cameron EE, Bachman KE, Myohanen S, Herman JG, Baylin SB. Synergy of demethylation and histone deacetylase inhibition in the re-expression of genes silenced in cancer. Nat Genet. 1999; 21:103-107. [PubMed: 9916800]

15. Ou JN, Torrisani J, Unterberger A, Provencal N, Shikimi K, Karimi M, Ekstrom TJ, Szyf M. Histone deacetylase inhibitor trichostatin a induces global and gene-specific DNA demethylation in human cancer cell lines. Biochem Pharmacol. 2007; 73:1297-1307. [PubMed: 17276411]

16. Wu LP, Wang X, Li L, Zhao Y, Lu S, Yu Y, Zhou W, Liu X, Yang J, Zheng Z, Zhang H, Feng J, Yang Y, Wang H, Zhu WG. Histone deacetylase inhibitor depsipeptide activates silenced genes through decreasing both cpg and h3k9 methylation on the promoter. Molecular and cellular biology. 2008; 28:3219-3235. [PubMed: 18332107]

17. Xiong Y, Dowdy SC, Podratz KC, Jin F, Attewell JR, Eberhardt NL, Jiang SW. Histone deacetylase inhibitors decrease DNA methyltransferase-3b messenger rna stability and downregulate de novo DNA methyltransferase activity in human endometrial cells. Cancer Res. 2005; 65:2684-2689. [PubMed: 15805266]

18. Yang X, Phillips DL, Ferguson AT, Nelson WG, Herman JG, Davidson NE. Synergistic activation of functional estrogen receptor (er)-alpha by DNA methyltransferase and histone deacetylase inhibition in human er-alpha-negative breast cancer cells. Cancer Res. 2001; 61:7025-7029. [PubMed: 11585728]

19. Peng DF, Razvi M, Chen H, Washington K, Roessner A, Schneider-Stock R, El-Rifai W. DNA hypermethylation regulates the expression of members of the mu-class glutathione s-transferases and glutathione peroxidases in barrett's adenocarcinoma. Gut. 2009; 58:5-15. [PubMed: 18664505] 
20. Lee OJ, Schneider-Stock R, McChesney PA, Kuester D, Roessner A, Vieth M, Moskaluk CA, ElRifai W. Hypermethylation and loss of expression of glutathione peroxidase-3 in barrett's tumorigenesis. Neoplasia. 2005; 7:854-861. [PubMed: 16229808]

21. Deng D, El-Rifai W, Ji J, Zhu B, Trampont P, Li J, Smith MF, Powel SM. Hypermethylation of metallothionein-3 cpg island in gastric carcinoma. Carcinogenesis. 2003; 24:25-29. [PubMed: 12538345]

22. Schneider BG, Peng DF, Camargo MC, Piazuelo MB, Sicinschi LA, Mera R, Romero-Gallo J, Delgado AG, Bravo LE, Wilson KT, Peek RM Jr, Correa P, El-Rifai W. Promoter DNA hypermethylation in gastric biopsies from subjects at high and low risk for gastric cancer. International journal of cancer.

23. Eads CA, Lord RV, Wickramasinghe K, Long TI, Kurumboor SK, Bernstein L, Peters JH, DeMeester SR, DeMeester TR, Skinner KA, Laird PW. Epigenetic patterns in the progression of esophageal adenocarcinoma. Cancer Res. 2001; 61:3410-3418. [PubMed: 11309301]

24. Clement G, Braunschweig R, Pasquier N, Bosman FT, Benhattar J. Alterations of the wnt signaling pathway during the neoplastic progression of barrett's esophagus. Oncogene. 2006; 25:3084-3092. [PubMed: 16407829] 

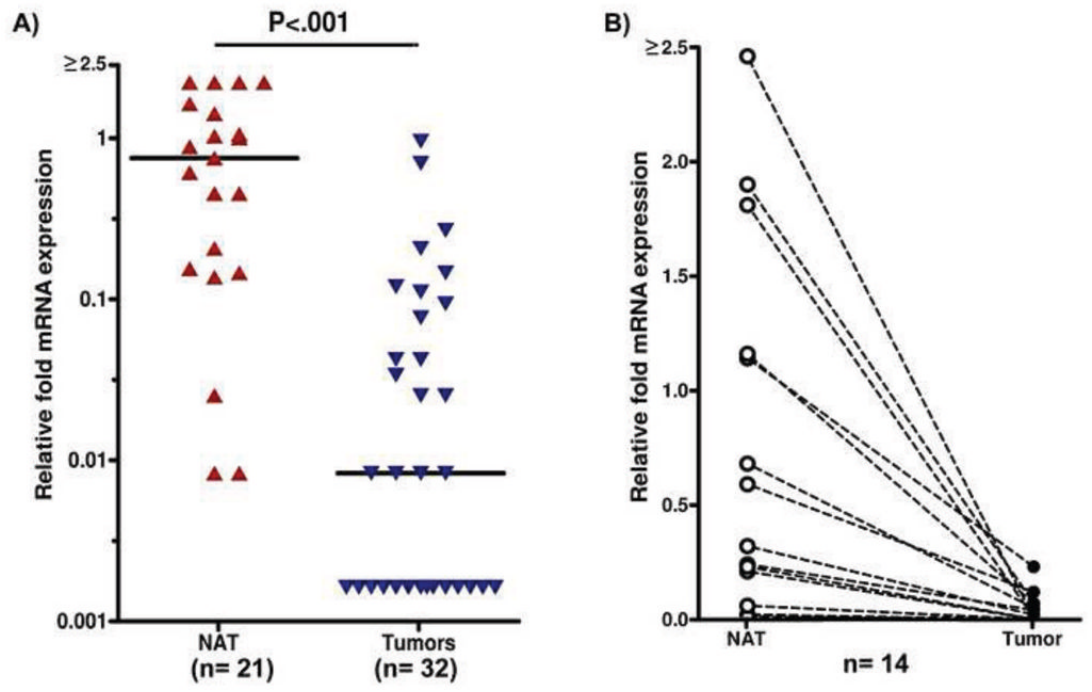

Figure 1. Comparison of SST mRNA expression levels in Normal vs Tumor

A) The expression of SST in normal mucosa $(n=21)$ and gastric carcinomas $(n=32)$ was determined by quantitative real-time RT-PCR. B) Expression of SST in 14 representative tumor samples and their corresponding normal samples from the same patients were analyzed side by side for comparison. 

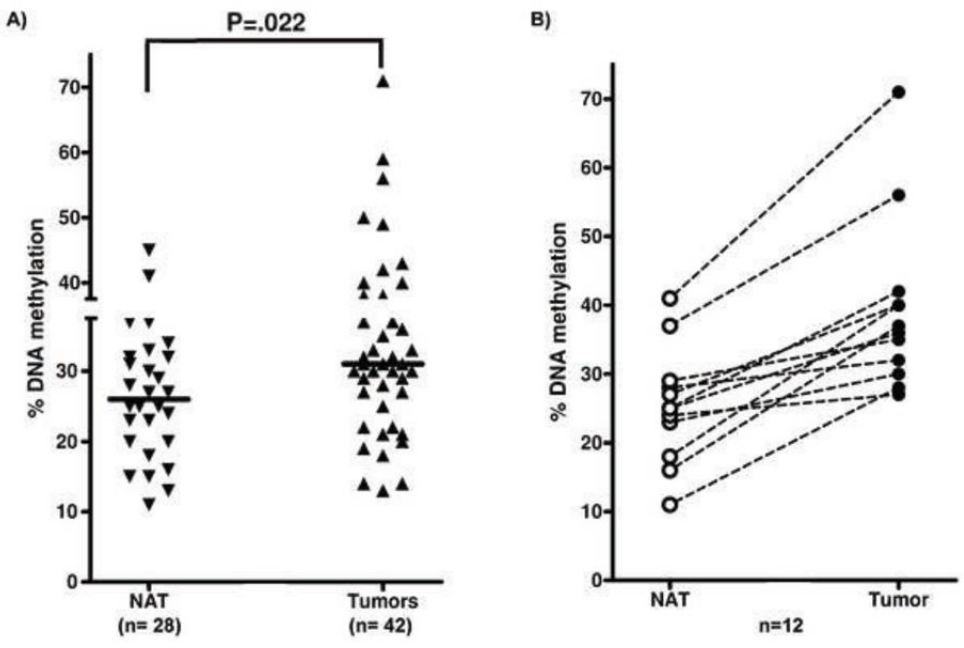

Figure 2. Comparison of DNA methylation levels

A) The percentage of promoter DNA methylation of the SST gene was determined by quantitative bisulfite pyrosequencing (Biotage). The horizontal bars locate the mean levels of DNA methylation. The statistical analysis of DNA methylation levels were determined by t-test. The tumors (gastric carcinomas) were compared to normal samples. B) Methylation levels of 12 representative matching normal and tumor samples. 


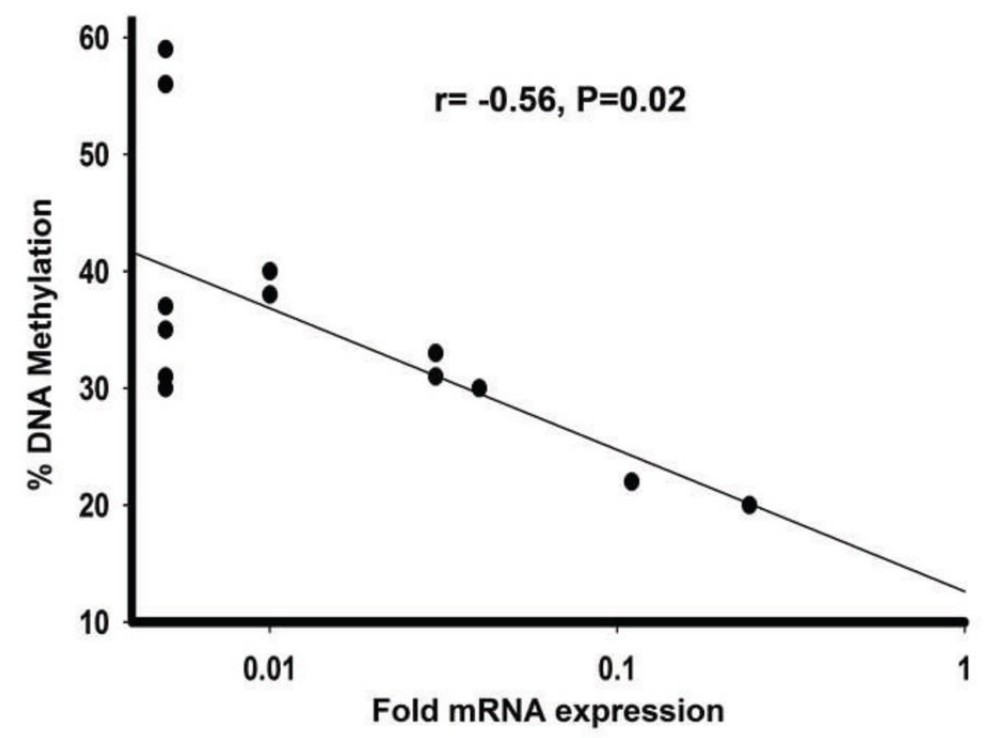

Figure 3. A correlation analysis between DNA methylation and gene expression levels A) The Spearman correlation analysis between the DNA methylation level and mRNA expression fold in SST gene. A significant inverse correlation was identified between promoter DNA methylation and mRNA expression fold for SST (coefficient $\mathrm{r}=-0.56$, $\mathrm{p}=0.02)$. 
A)

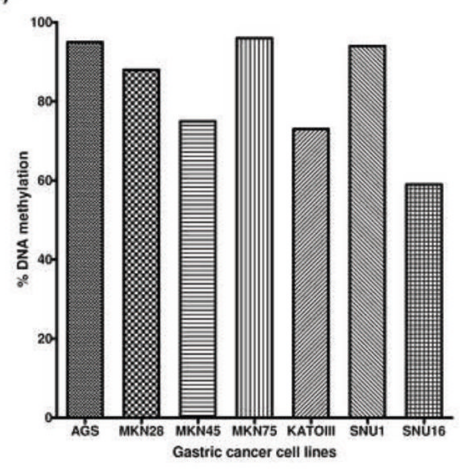

B)

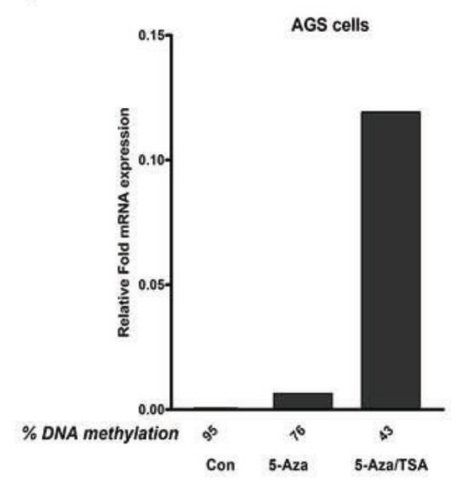

Figure 4. Analysis of methylation and expression levels of SST in gastric cancer cell lines A) High levels of DNA methylation of SST were detected in gastric cancer cell lines (AGS, MKN28, MKN45, MKN75, KATOIII, SNU1, and SNU16). B) Treatment of AGS gastric cancer line with 5-aza-dC and TSA led to a decrease in promoter DNA methylation and mRNA re-expression of SST. 